\title{
Investigation of Millimeter Wave Extended Interaction Oscillation using Improved Pseudospark-sourced Electron Beams
}

\author{
H. Yin ${ }^{1}$, L. Zhang ${ }^{1}$, W. He ${ }^{1}$, G. Shu ${ }^{1}$, J. Zhao ${ }^{2}$, Y. Yin ${ }^{3}$, K. Ronald ${ }^{1}$, A.D.R. Phelps ${ }^{1}$, and A.W. Cross ${ }^{1}$ \\ ${ }^{1}$ Department of Physics, SUPA, University of Strathclyde, Glasgow, G4 0NG, Scotland, UK \\ ${ }^{2}$ High Voltage Division, School of Electrical Engineering, Xi'an Jiaotong University, Xi' an, 710049, China \\ ${ }^{3}$ School of Physical Electronics, University of Electronic Science \& Technology of China, Chengdu, China
}

\begin{abstract}
This article presents the investigation of millimeter wave extended interaction oscillation using both pencil and sheet-shaped pseudospark (PS)-sourced electron beams. A W-band (75-110 GHz) pencil beam extended interaction oscillator (EIO) was designed and constructed. In the first experiment the output power of $38 \mathrm{~W}$ was achieved from a four-gap PS discharge operating at $30.5 \mathrm{kV}$. PS-sourced beam generation was further improved by combining a single-gap PS structure with an integrated post-acceleration section in order to enhance the beam quality and increase the output power of the EIO to $200 \mathrm{~W}$. The study of a PS-sourced sheet beam EIO structure will also be presented.
\end{abstract}

\section{INTRODUCTION AND BACKGROUND}

$\mathrm{M}$ ILLIMETER wave sources in the frequency range from 0.1 to $1 \mathrm{THz}$ have received increasing interest in recent years because of their many exciting applications such as in electron spin resonance spectroscopy, remote imaging, radar and $5 \mathrm{G}$ high speed mobile wireless communications. To date, vacuum electronic technology still remains the main method to achieve millimeter wave radiation of high power up to the kilowatt level, while conventional optical and electronic technologies are struggling to provide good frequency bandwidth at even rather moderate power levels. High quality intense electron beams have been crucial in vacuum electronic devices, thus the PS discharge has attracted a lot of attention recently as a promising source of high quality high intensity electron beam pulses with the beam current density up to $10^{6} \mathrm{Am}^{-2}$ and brightness up to $10^{12} \mathrm{Am}^{-2} \mathrm{rad}^{-2}$ [1-3]. This is because a PS-sourced electron beam has the ability to self-focus due to the unique structure and the formation of an ion channel generated by the beam front. Simulations have shown that the PS-sourced electron beam can propagate within background plasma without any applied guiding magnetic field. This makes it an excellent electron beam source for desirable compact millimeter wave devices. Among various vacuum electronic devices, the EIO as a linear beam vacuum device has gained considerable attention as a promising millimeter wave oscillation source due to its high gain per unit length and compact configuration [4-7].

In this paper, we use a PS-sourced electron beam (PS-EB) instead of the conventional electron beam produced by a thermionic cathode to drive an EIO to achieve more compact devices. To achieve better beam quality, the PS-sourced electron beam is also investigated with post-acceleration to reduce its beam energy spread [8-10]. To achieve higher output power, a sheet beam EIO structure is further studied with a PS-sourced sheet beam [11].

\section{EXPERIMENTS AND RESULTS}

Fig.1 shows the first pencil beam EIO experimental setup with the four-gap PS discharge configuration. When the discharge was operating at $30.5 \mathrm{kV}$, an output power of $38 \mathrm{~W}$ was measured.

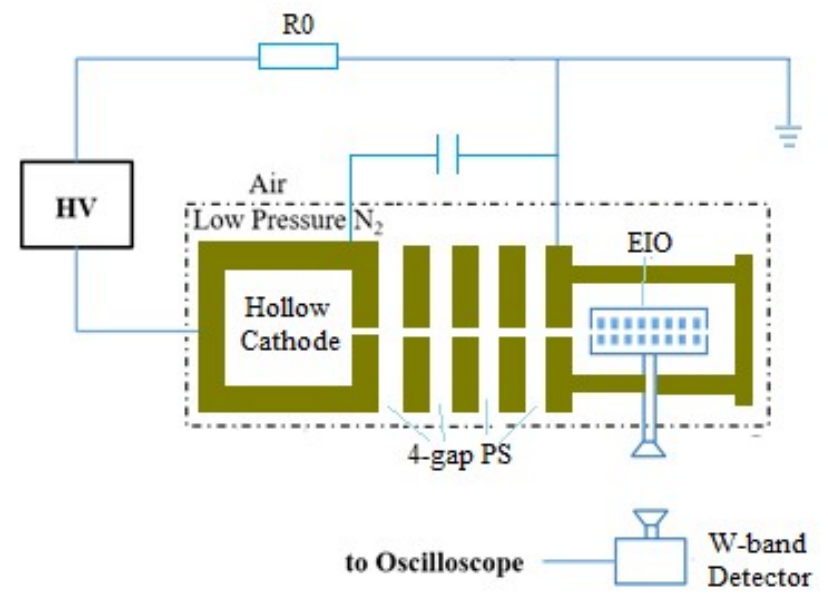

Fig. 1 Experimental setup of the pencil beam EIO driven by a PS-sourced electron beam

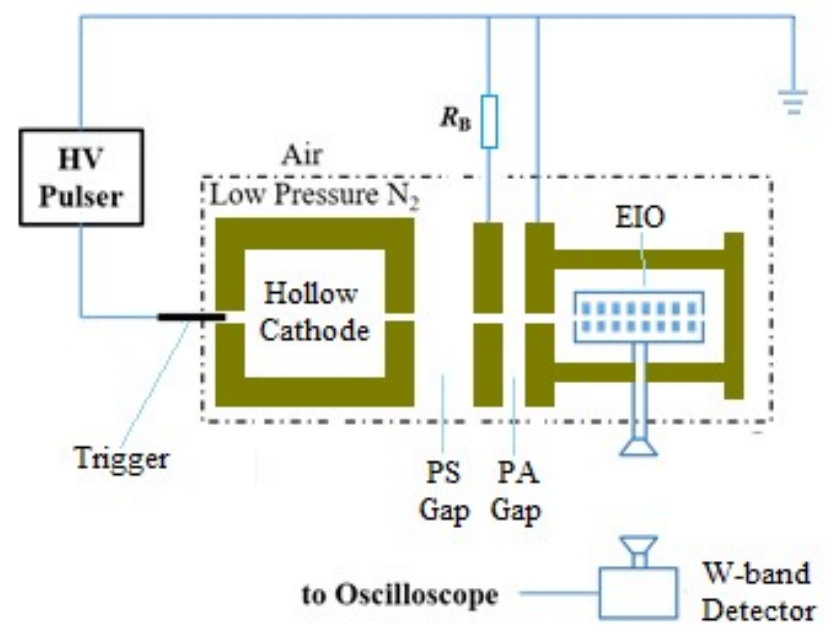

Fig. 2 Experimental setup of the pencil and sheet beam EIOs driven by a post-accelerated PS-sourced electron beam

Fig. 2 shows the experimental setup of a one-gap PS discharge combining with an integrated post-acceleration section to drive the same pencil beam EIO structure. The post-acceleration section is used to improve the PS beam quality by reducing beam energy spread. Fig.3 shows some measurements of the beam voltage and current, the image of the beam cross section, and the beam intensity analysis. Fig. 4 shows a typical time-correlated 
electron beam voltage, current pulse, the radiation pulse from the W-band EIO with this configuration, and an output power of up to $200 \mathrm{~W}$ was measured as compared with the configuration in [7] where the output power of only $38 \mathrm{~W}$ was achieved from a 4-gap PS discharge configuration.

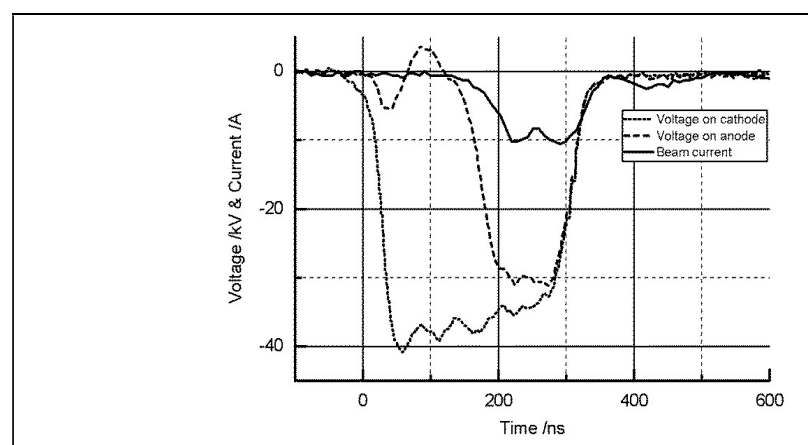

(a)

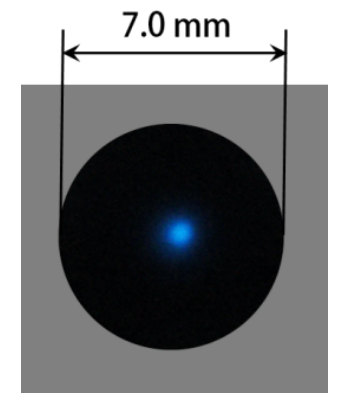

(b)

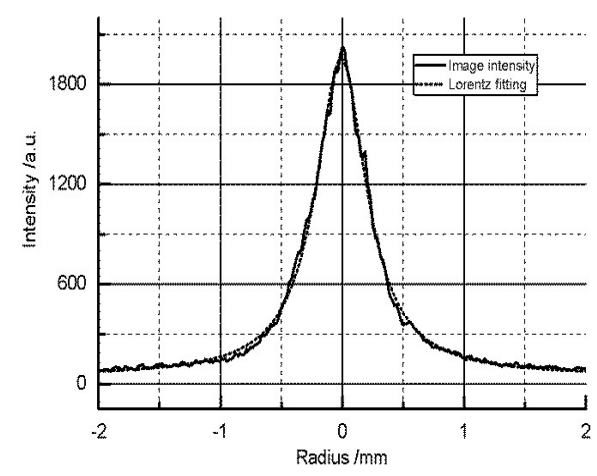

(c)

Fig.3 Typical waveforms of beam voltage and current (a), beam cross section image (b), and beam intensity along the radius of the beam(c)

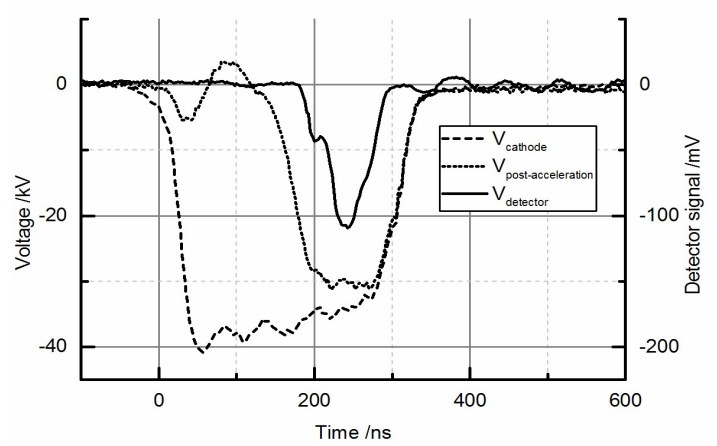

Fig.4 Typical time-correlated electron beam voltage, current pulse, the radiation pulse from the W-band EIO
With this improved configuration, further studies of a planar EIO with a PS sheet electron beam were conducted as shown in Fig.5. Preliminary experimental results have shown a much higher output power achieved from a W-band planar EIO. Further studies of a planar EIO with a PS sheet electron beam will be conducted using this improved configuration and a higher frequency $200 \mathrm{GHz}$ EIO structure in the near future.

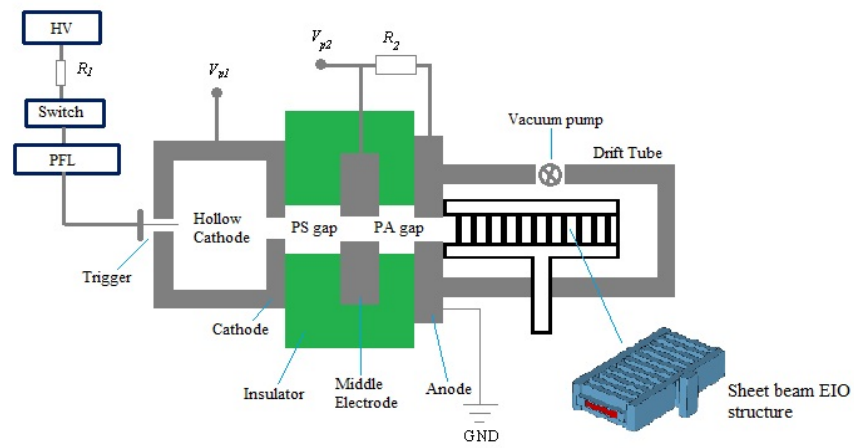

Fig. 5 Experimental setup of the EIO driven by a PS-sourced electron beam

\section{REFERENCES}

[1] D. Bowes, H. Yin, W. He, A.W. Cross, K. Ronald, A.D.R. Phelps, D. Chen, P. Zhang, X. Chen and D. Li, "Visualization of a Pseudospark-Sourced Electron Beam", IEEE Trans. Plasma Science, vol. 42, no. 10, pp2826-2827, Oct. 2014.

[2] H. Yin, W. He, A.W. Cross, A.D.R. Phelps, and K. Ronald, "Single-gap pseudospark discharge experiments," J. Appl. Phys., vol. 90, pp. 3212-3218, Oct . 2001.

[3] J. Zhao, H. Yin, L. Zhang, G. Shu, W. He, J. Zhang, Q. Zhang, A. D. R. Phelps, and A. W. Cross, "Influence of the electrode gap separation on the pseudospark-sourced electron beam generation," Phys. Plasmas, vol. 23, 073116, July 2016.

[4] H. Yin, A. W. Cross, W. He, A. D. R. Phelps, K. Ronald, D. Bowes, C.W. Roberson "Millimeter wave generation from a pseudospark-sourced electron beam".,Phys. Plasmas 16 (2009).

[5] W. He, L. Zhang, D. Bowes, H. Yin, K. Ronald, A. D. R. Phelps, and A. W. Cross, "Generation of broadband terahertz radiation using a backward wave oscillator and pseudospark-sourced electron beam," Appl. Phys. Lett., vol. 107, no. 13, 133501, Sept. 2015.

[6] Y. Yin, W. He, L. Zhang, et al., "Preliminary design and optimization of a G-band extended interaction oscillator based on a pseudospark-sourced electron beam," Phys. Plasmas, vol. 22, no. 7, pp. 073102-1-073102-6, Jul. 2015.

[7] Y. Yin, W. He, L. Zhang, H. Yin, C. W. Robertson and A. W. Cross, "Simulation and Experiments of a W-band Extended Interaction Oscillator based on a pseudospark-sourced electron beam," IEEE Trans. Electron Devices, vol. 63, no. 1, pp. 512 - 516, Jan. 2016.

[8] J. Zhao, H. Yin, L. Zhang, G. Shu, W. He, Q. Zhang, A. D. R. Phelps, and A. W. Cross, "Advanced post-acceleration methodology for pseudospark-sourced electron beam", Phys. Plasmas. vol. 24, no. 2, 023105 , Feb. 2017.

[9] J. Zhao, H. Yin, L. Zhang, G. Shu, W. He, Q. Zhang, A. D. R. Phelps, and A. W. Cross, " Study of the beam profile and position instability of a post-accelerated pseudospark-sourced electron beam", Phys. Plasmas. vol. 24, 033118, March. 2017.

[10] J. Zhao, H. Yin, L. Zhang, W. He, Q. Zhang, A. D. R. Phelps, and A. W. Cross, " Experiments on W-band extended interaction oscillator with pseudospark sourced post-accelerated electron beam", Phys. Plasmas. In press, May. 2017

[11] G. Shu, W. He, L. Zhang, Y. Yin, J. P. Zhao, A. W. Cross, A. D. R. Phelps, "Study of a $0.2 \mathrm{THz}$ extended interaction oscillator driven by a pseudospark-sourced sheet electron beam," IEEE Trans. Electron Devices, vol. 63, no. 12, pp.4955-4960, Dec. 2016 